\title{
Liquid Crystal-Based Hydrophone Arrays
}

\section{Zourab BRODZELI ${ }^{*}$, Leonardo SILVESTRI ${ }^{1}$, Andrew MICHIE ${ }^{2}$, Vladimir G. CHIGRINOV ${ }^{3}$, Qi GUO ${ }^{3}$, Eugene P. POZHIDAEV ${ }^{4}$, Alexei D. KISELEV ${ }^{5}$, and Francois LADOUCEUR ${ }^{1}$}

\author{
${ }^{1}$ School of Electrical Engineering and Telecommunications, UNSW, Sydney NSW 2052, Australia \\ ${ }^{2}$ Smart Digital Optics Pty. Ltd., Suite 145 National Innovation Centre 4 Cornwallis Street Australian Technology Park \\ Eveleigh, NSW, Australia 2015 \\ ${ }^{3}$ CDR, Department of Electrical and Electronic Engineering, The Hong Kong University of Science and Technology, \\ Clear Water Bay, Kowloon, Hong Kong, China \\ ${ }^{4}$ P. N. Lebedev Physics Institute of Russian Sciences, Leninsky Prospect 53, 117924 Moscow, Russia \\ ${ }^{5}$ Institute of Physics of National Academy of Sciences of Ukraine, Prospekt Nauki 46, 03028 Kÿ̈v, Ukraine \\ *Corresponding author: Zourab BRODZELI_ E-mail: zourab.brodzeli@unsw.edu.au
}

\begin{abstract}
We describe a fiber optic hydrophone array system that could be used for underwater acoustic surveillance applications (e.g. military, counter terrorist, and customs authorities in protecting ports and harbors), offshore production facilities or coastal approaches as well as various marine applications. In this paper, we propose a new approach to underwater sonar systems using the voltage-controlled liquid crystals and simple multiplexing method. The proposed method permits measurement of sound under water at multiple points along an optical fiber using the low cost components and standard single mode fiber, without complex interferometric measurement techniques, electronics or demodulation software.
\end{abstract}

Keywords: Ferroelectric liquid crystal, electrooptic response, hydrophone, sonar, deformed helix ferroelectric liquid crystal, fiber Bragg grating

\section{Introduction}

Today's underwater sound detection and mapping technology is based on a complex, large and expensive electrical approach [1]. The search for an optical version of the electrical sensors used today has been pursued with increasing vigor over the past decade due to the emergence of a viable fiber-based technology and with accompanying low-cost optical components.

The idea of using an optical fiber as a hydrophone was first published by Bucaro [2]; since then, various fiber-based sensors have been developed [3-7]. These rely either on (1) some interferometric approach including the use of fiber coils and distributed feedback (DFB) fiber-lasers or (2) one of a number of intensity modulation mechanisms, the latter involving physical motion or mechanical effects such as micro bending. However, each of these approaches must overcome its own set of problems - including complexity, cost or sensitivity - before truly practical sensors can be

Received: 12 June 2012 / Revised version: 18 June 2012

(C) The Author(s) 2012. This article is published with open access at Springerlink.com 
produced.

In this paper, we propose an approach based on liquid crystal cells as variable, broadband reflectors in combination with ceramic hydrophones, standard single mode fiber, and fiber Bragg gratings (FBGs). The proposed method does neither rely on complex interferometric measurement techniques, electronics nor software for demodulating the output signals. This system uses simple demultiplexing of the output light signal based on both amplitude and frequency of light in predefined channels, which are directly linked to the amplitude and frequency of the sound signals measured under water.

\section{Sensing system}

The proposed sensing system, illustrated in Fig. 1, consists of two major parts: a sensor head (SH), monitoring pressure changes under water, and a multiplexing system, allowing measurement at distributed points in space by sharing a single broadband source (BBS) with multiple sensor heads.

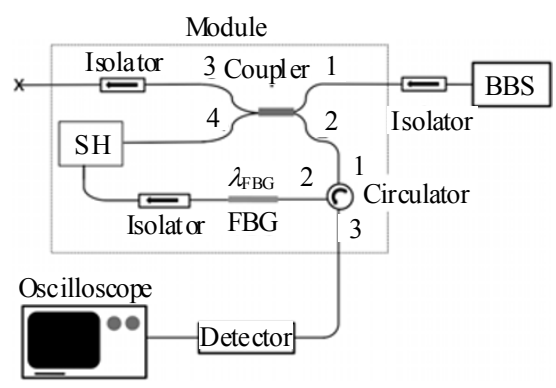

(a)

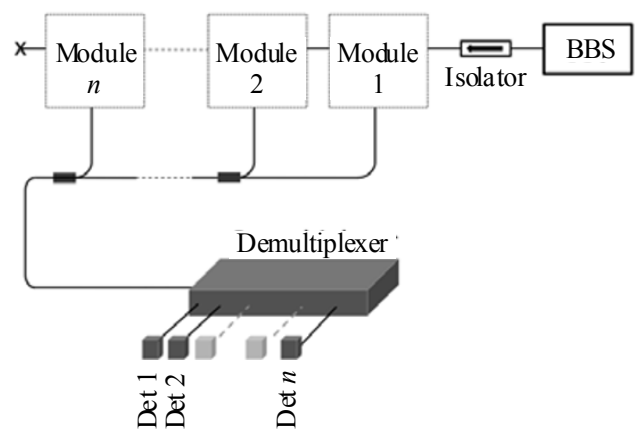

(b)

Fig. 1 Multiplexing system.
The source provides a broadband unpolarized optical signal to multiple modules, each routing part of the light to the corresponding sensor head. Each one in turn reflects light, modulating its intensity according to the measured pressure, and an FBG ensures that only a specific wavelength (channel) is transmitted to the demultiplexer (DM) from each module. The demultiplexer gathers all the channels reflected from the SHs and directs each channel into the monitoring devices (photo-detectors).

\subsection{Sensor head}

The purpose of the sensor head is to monitor pressure changes under water and to transduce them linearly into a variation of intensity of the optical signal. The sensor head is a hybrid device based on the combination of well-established technologies, namely fiber optics, piezoelectric materials (PZT), and liquid crystals (LC). It utilizes the excellent wave guiding properties of optical fibers to leverage the high electro-optic and piezoelectric constants of LCs and PZTs. The head is composed of a liquid crystal cell, a polarization maintaining (PM) fiber, an in-line polarizer, a standard single-mode fiber (e.g. SMF 28), and a PZT hydrophone equipped with an optically powered amplifier (Fig. 2).

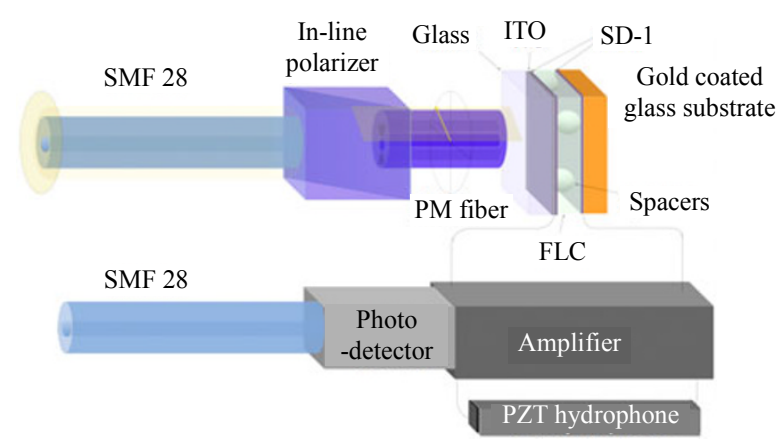

Fig. 2 Sensor head.

The liquid crystal cell itself consists of two sandwiched glass substrates, with their inner surfaces coated with indium tin oxide (ITO) and gold, respectively. Both coatings are used as electrodes thus enabling the creation of electrical 
fields between the glass substrates. The gold coating is also used as a broadband reflector, allowing the operation of the cell in reflection. The gap between the substrates is filled with a short pitch ferroelectric liquid crystal (FLC) with its helix axis lying on the plane defined by the substrate surface and perpendicular to the electric field (Fig. 3). In this configuration, the cell operates in deformed helix ferroelectric (DHF) mode [8], featuring a linear dependence of the cell reflectivity on the applied electrical field under crossed or parallel polarisers [9, 10]. The behavior of the DHF structure in an electric field has been fully characterized and will be discussed in greater detail below.

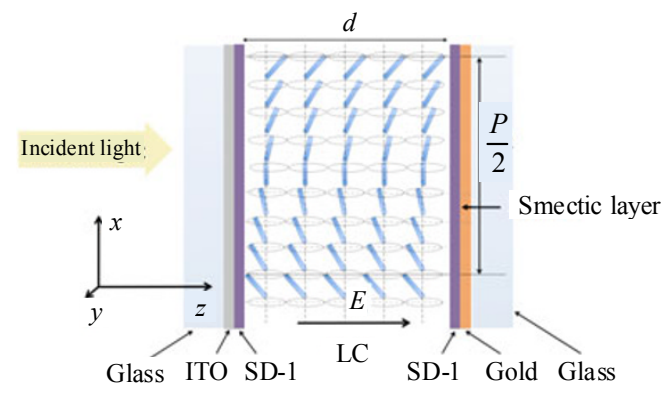

Fig. 3 Liquid crystal cell.

The principle of operation of the sensor head is as follows. Unpolarized light coming from the broadband source is guided by a standard single mode fiber, polarized by an in-line polarizer at an angle $\beta$ to the liquid crystal helix axis and supplied to the cell using a polarization maintaining (PM) fiber. Polarized light impinges at normal incidence on the first substrate, passes through the liquid crystal, reflects off the gold-coated mirror back into the same layer and is collected by the fiber. The presence of an electric field directed along the light propagation direction, induces a change in the birefringence of the liquid crystal and therefore affects the measured reflectance. The proposed configuration corresponds to a parallel polarizer/analyzer geometry and allows to transduce the applied voltage into a modulation of the intensity of the reflected light, which is then detected by the photo-diode.

The electric field required to switch the cell is around a few volts (typically $5 \mathrm{~V}$ to $10 \mathrm{~V}$ ) per $\mu \mathrm{m}$ $(5 \mathrm{kV} / \mathrm{m}$ to $10 \mathrm{kV} / \mathrm{m})$ and is thus much higher than the field directly created by the PZT actuator (ceramic hydrophone). The sensitivity of modern ceramic actuators is $-164 \mathrm{~dB}$ at $1 \mathrm{~V} / \mu \mathrm{Pa}$. The response of this ceramic hydrophone to sea state zero (SS0) and to various underwater noises ranges between $0.1 \mu \mathrm{V}$ and $0.6 \mathrm{~V}$. Thus, some field amplification is required. This amplification can be accomplished using an optically powered amplifier. The optical signal transmitted through an FBG with a wavelength not satisfying the Bragg condition could be used for powering the amplifier.

\subsection{Multiplexing}

The multiplexing system presented in this work was developed using modules consisting of a $3-\mathrm{dB}$ $2 \times 2$ bi-directional coupler, a circulator incorporating an FBG and isolators in the configuration shown in Fig. 1(a). Port 4 of the coupler is connected to the sensor head, and port 1 is connected to the broadband light source via an isolator. Any change in reflectivity at the sensing end would then result in a variation of the signal intensity reflected back to port 2. Port 2 of the coupler is connected to the circulator via port 1, thus enabling the reflected sensing signal to reach the FBG connected to port 2 of the circulator. The FBG is used to selectively reflect back to port 3 of the circulator (signal detection end) the part of the broadband signal matching the FBG's narrow spectral band $\left(\lambda_{\mathrm{FBG}}\right)$. As a result, information about the value of the electrical field or voltage can be retrieved by monitoring the relative intensity of the signal reflected back from the sensor head to the FBG and then to the detector or the optical spectrum analyser (OSA). The broadband signal transmitted through the FBG is 
connected to the photo-detector via the isolator. This part of optical signal is used to harvest energy to power the amplifier connected to the PZT hydrophone. This sensing module allows multiplexing capability by directly connecting a similar module with port 3 of the coupler, as depicted in Fig. 1(b). An optical isolator is used to avoid crosstalk between the modules.

\subsection{Modeling of the liquid crystal cell}

In this section, we describe in more detail the behavior of the DHF mode when the cell is operated in reflection. Adopting a polarization-gratings approach, Kiselev et al. [11] have shown that when the helix pitch, $P$, is smaller than the incident wavelength, $\lambda$, the liquid crystal can be described as a uniform biaxial material. In our case $P=200 \mathrm{~nm}$ and $\lambda=633 \mathrm{~nm}$, so that we feel justified in adopting the same approach. In the geometry illustrated in Fig. 3, where the helix pitch is along $x$, smectic layers are in the $y-z$ plane and the electric field is along $z$, the effective dielectric tensor at small fields can be written as

$$
\varepsilon\left(\alpha_{E}\right)=\left(\begin{array}{ccc}
\varepsilon_{x x}\left(\alpha_{E}\right) & \gamma_{x y} \alpha_{E} & 0 \\
\gamma_{x y} \alpha_{E} & \varepsilon_{y y}\left(\alpha_{E}\right) & 0 \\
0 & 0 & \varepsilon_{z z}\left(\alpha_{E}\right)
\end{array}\right)
$$

with

$$
\left\{\begin{array}{l}
\varepsilon_{x x}\left(\alpha_{E}\right)=\varepsilon_{x x}^{(0)}+\gamma_{x x} \alpha_{E}^{2} \\
\varepsilon_{y y}\left(\alpha_{E}\right)=\varepsilon_{y y}^{(0)}+\gamma_{x x} \alpha_{E}^{2} . \\
\varepsilon_{z z}\left(\alpha_{E}\right)=\varepsilon_{y y}^{(0)}-\gamma_{x x} \alpha_{E}^{2}
\end{array}\right.
$$

The effective dielectric tensor $\varepsilon$ depends on the electric field through the electric field parameter $\alpha_{E}$, which is proportional to the ratio of the applied and the critical electric field, $E / E_{\mathrm{C}}$. Since both the model and the device only work for fields much smaller than the critical field, $E<<E_{\mathrm{C}}$, in (1) and (2), we expand each term to the second order in $\alpha_{E}$, following [11]. All the coefficients appearing in (1) and (2) can be calculated as a function of the liquid crystal parameters, as decribed in [11]. In the $x^{\prime} y^{\prime} z^{\prime}$ reference system of the liquid cristal principal axes, which rotates with the electric field, the effective dielectric tensor can be written in the diagonal form as

$$
\varepsilon^{\prime}\left(\alpha_{E}\right)=\left(\begin{array}{ccc}
\varepsilon_{+}\left(\alpha_{E}\right) & 0 & 0 \\
0 & \varepsilon_{-}\left(\alpha_{E}\right) & 0 \\
0 & 0 & \varepsilon_{z z}\left(\alpha_{E}\right)
\end{array}\right)
$$

where

$$
\begin{aligned}
\varepsilon_{ \pm}\left(\alpha_{E}\right)= & \frac{\varepsilon_{x x}\left(\alpha_{E}\right)+\varepsilon_{y y}\left(\alpha_{E}\right)}{2} \pm \\
& \pm \frac{\varepsilon_{x x}\left(\alpha_{E}\right)-\varepsilon_{y y}\left(\alpha_{E}\right)}{2} \sqrt{1+\tan ^{2}\left[2 \Omega\left(\alpha_{E}\right)\right]}
\end{aligned}
$$

with

$$
\Omega\left(\alpha_{E}\right)=\frac{1}{2} \arctan \left[\frac{2 \gamma_{x y}}{\varepsilon_{x x}\left(\alpha_{E}\right)-\varepsilon_{y y}\left(\alpha_{E}\right)} \alpha_{E}\right],
$$

the latter being the angle between the principal axis with the highest refractive index and the helix axis. In order to calculate the structure reflectance, we place ourselves in the optical axis frame of reference, where the liquid crystal effective dielectric tensor is diagonal and assume that gold is a perfect mirror, i.e. that its refractive index modulus is much larger than all the liquid crystal refractive indices. By using a transfer matrix approach, we can calculate the reflection coefficient for the structure in Fig. 3 in the case of normal incident light along $z$. Let us assume that the incident light is linearly polarized at an angle $\beta$ with respect to the helix axis $x$ and that LC refractive indices are much larger than the in-plane refractive index difference:

$$
\Delta n\left(\alpha_{E}\right)=\sqrt{\varepsilon_{+}\left(\alpha_{E}\right)}-\sqrt{\varepsilon_{-}\left(\alpha_{E}\right)} .
$$

We find that the reflectance measured with crossed polarizer/analyzer, is given by

$$
R_{\perp}\left(\alpha_{E}\right)=\sin ^{2}\left[d \frac{2 \pi}{\lambda} \Delta n\left(\alpha_{E}\right)\right] \sin ^{2}\left[2 \beta-2 \Omega\left(\alpha_{E}\right)\right](7)
$$

while the reflectance with parallel polarizer/analyzer is

$$
R_{\|}\left(\alpha_{E}\right)=1-R_{\perp}\left(\alpha_{E}\right) .
$$


With (5)-(8), we can calculate the reflectance with the crossed or parallel polarizer/analyzer for each value of the applied electrical field. In the experiments, the voltage $V$ across the LC cell is known, so that we can write

$$
\alpha_{E}=\gamma_{E} V / d
$$

where we introduce the proportionality constant $\gamma_{E}$, which is usually treated as a parameter of the theory and can be adjusted to fit the experimental data.

\section{Experimental results}

\subsection{Fabrication}

The liquid crystal cells consist of two parallel glass substrates separated by a distance $d$ using spacers (beads) of control sizes: $5 \mu \mathrm{m}$ for the bulk approaches and $10 \mu \mathrm{m}$ for the fiber optic one. One substrate is coated with ITO and the other with gold thus forming two electrodes one of which also acts as a broadband reflector, which allows the cell to be used in reflection.

P. N. Lebedev at the Physical Institute of Russian Academy of Sciences developed the DHF liquid crystal (tagged as FLC-576) used in the cell with an helix pitch $P_{0}=200 \mathrm{~nm}$ [11]. This particular liquid crystal mixture was chosen due to its short helix pitch yielding low scattering of light and allowing DHF mode of operation as $P_{0}<<d$ [4]. Importantly, the planar alignment of the liquid crystals was achieved using a photo-alignment method [12]. In this approach, both inner surfaces of the substrates were spin-coated with a photo-aligning substance (azo-benzene sulfuric dye SD-1 dried at $155^{\circ} \mathrm{C}$ ), and polarized ultraviolet (UV) irradiation $\left(6 \mathrm{~mW} / \mathrm{cm}^{2}\right.$ at $\left.365 \mathrm{~nm}\right)$ of the azo-dye films at normal incidence was used to induce anisotropy.

\subsection{Experimental setup}

Two experimental setups were built to test the theoretical model and characterize the sensor head
[Figs. 4(a) and 4(b)]. The purpose of the measurement was to test the model and to adjust the parameters of the liquid crystal cells used in our experiments. Linearly polarized light from a $\mathrm{He}-\mathrm{Ne}$ laser at $632 \mathrm{~nm}$ at normal incidence was used; the light reflected back from the golden inner surface of the cell was transmitted through the non-polarizing beam-splitter, which directed $50 \%$ of light onto the photo-detector. The analyzer was installed and aligned orthogonally to the polarization of the incident light in front of the photo-detector. An aperture was used to block light scattered from the cell, and the cell was placed on a rotation stage allowing us to vary the angle between polarization of the incident light and the helix axis.

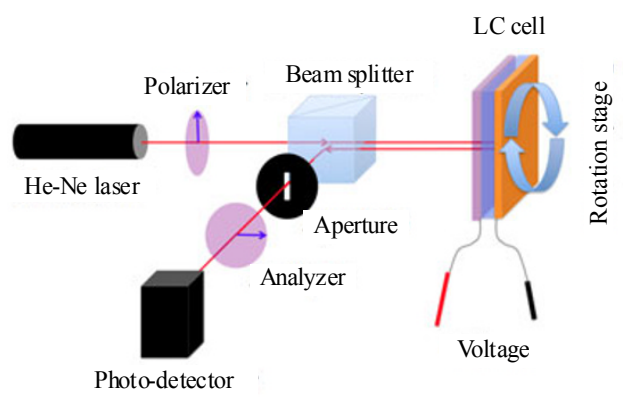

(a)

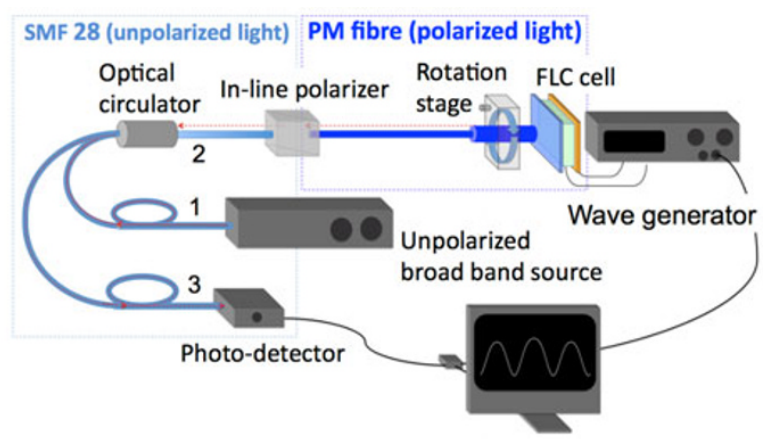

(b)

Fig. 4 Schematic representation of the experimental setup using (a) bulk optics and (b) fiber optics.

The principle of operation of the cell as a voltage sensor was tested using the fiber optic setup presented in Fig. 4(b). The unpolarized broadband optical signal emitted (BBS15/16 AFC Technologies Inc.) was launched into port 1 of the optical 
circulator (6015-3Thorlabs Inc.). The circulator directed the light into port 2, which was connected to the in-line polarizer, transforming the unpolarized light into linearly polarized light. The output of the in-line polarizer, containing the collimated polarization maintaining fiber (C-PM-15 AFW Technology Pty. Ltd.), was directly butted onto the glass substrate of the cell and delivered highly polarized light. The cell was operated in reflection and could be described as a variable reflector. The angle $\beta$ between the polarization of the incident light and the helix axis was adjusted by rotating the collimator placed inside the rotation stage. The polarized light propagating through the birefringent liquid crystal split into two components propagating at different speeds along the ordinary and extraordinary optical axes. At the output of the cell, the two components interfered, resulting in a rotated polarization state. The polarization maintaining fiber, together with the in-line polarizer at the output of the cell, transformed this output polarization state into a variation of the intensity. The optical circulator directed the optical signal from the cell into port 3, which was connected to the variable gain photo-detector used to monitor the variation of the intensity of the light reflected as a function of the voltage. The signal generator (DS340 Stanford Research Systems Inc.) was used to simulate the output of the piezoelectric hydrophone and generate the variable voltage applied to the cell. The applied voltage had a sinusoidal form with frequency variable from a few $\mathrm{Hz}$ to $10 \mathrm{kHz}$. Computer controlled signal generator and photo-detector were used allowing the logging of the cell's response.

\subsection{Results and discussion}

The cell was first characterized to show that the theoretical model could describe its operation using the setup presented in Fig. 4(a). Experimental data are shown in Fig. 5 and compared to the simulation results. Calculations have been performed as described in Section 2.3 using the formulas of [11] and the following parameters: the ordinary and extraordinary refractive indices $n_{\mathrm{o}}=1.5$ and $n_{\mathrm{e}}=1.69$, respectively, the tilt angle $\theta=32^{\circ}$ and $\gamma_{E}=0.44$. We also scaled the final reflectance by a factor 0.52 , in order to take into account the losses, mainly due to scattering of light inside the LC. We see that the model correctly describe the general behavior of the reflectance for different polarizations. In particular, the crossed polarizer reflectance at $V=0$ is predicted to be maximum for $\beta=45^{\circ}$ and to vanish for $\beta=0^{\circ}$. In both these cases, the dependence of the reflectance from the electric field is quadratic at small fields. We also point out that the reflectance is a periodic function of $\beta$ with a period of $90^{\circ}$ so that it is sufficient to consider polarizations in the range $-45^{\circ}<\beta<45^{\circ}$.

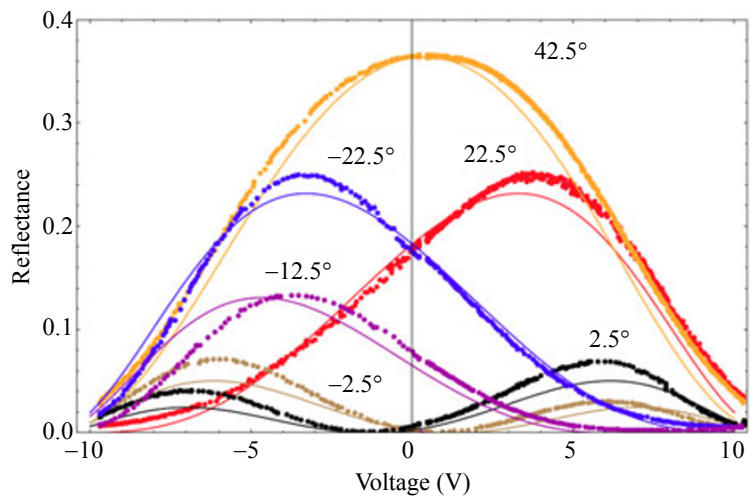

Fig. 5 Reflectance as a function of the applied voltage for various incident light polarizations $\beta$, as indicated [symbols represent experimental results, while solid lines have been calculated with formulas (5)-(7)].

It is now interesting to discuss under which conditions the reflectance is a linear function of the applied voltage. This is because in this régime, the cell could be used to convert a time-dependent electrical signal into an optical signal without distortion. Let us assume that the applied voltage has a sinusoidal time dependence, $V(t)=V_{0} \sin (\omega t)$ and that the frequency $\omega$ is low enough that the cell 
response can be considered within the static limit. A useful quantity to determine the optimal linear response is the THD, which can be expressed in decibels as

$$
\operatorname{THD}\left(\beta, V_{0}\right)=10 \log _{10}\left[\frac{P_{N}+\sum_{n=2}^{\infty} P_{n}\left(\beta, V_{0}\right)}{P_{1}\left(\beta, V_{0}\right)}\right]
$$

where $P_{N}$ is the noise, and $P_{n}$ is the power of the $n$th harmonic, calculated as the square of the $\sin (n \omega t)$ coefficient in the reflectance Fourier series. The lower the THD is, the more linear the response is.

We report in Fig. 6 the calculated THD for the

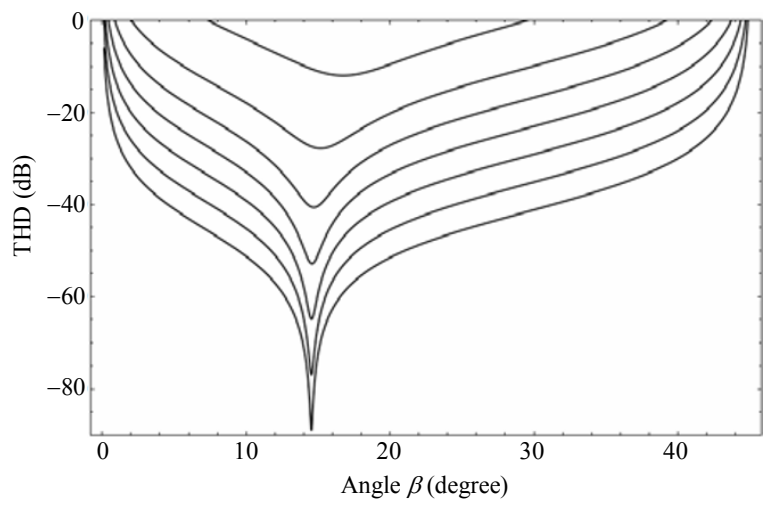

(a) cell used in the experiment at $P_{N}=0$. Each curve in Fig. 6(a) corresponds to a different value of $V_{0}$. The lowest THD corresponds to $\beta=14.5^{\circ}$, which is close to the value of $12^{\circ}$ observed experimentally. The THD decreases with a decrease in the amplitude of the applied voltage, which is illustrated in Fig. 6(b), where the two curves corresponding $\beta=12^{\circ}$ and $\beta=$ $14.5^{\circ}$ are presented. We notice that for small voltages and selected polarizations, the distortion can be very low opening up interesting sensing applications, in particularly the detection of sound waves.

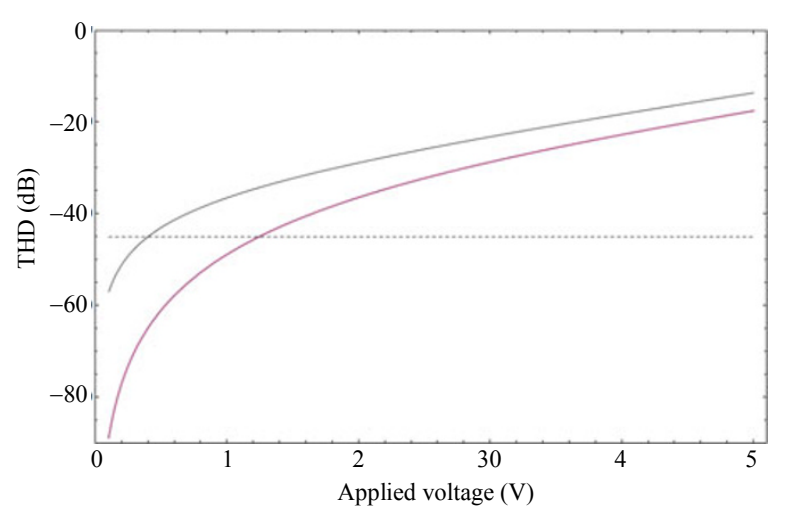

(b)

Fig. 6 Total harmonic distortion (THD): (a) calculated as a function of $\beta$ for different applied voltages ranging from $0.1 \mathrm{~V}$ (bottom) to $6.4 \mathrm{~V}$ (top) and (b) calculated as a function of the applied voltage at $\beta^{\circ}=12^{\circ}$ (top curve) and $\beta=14.5^{\circ}$ (bottom curve).

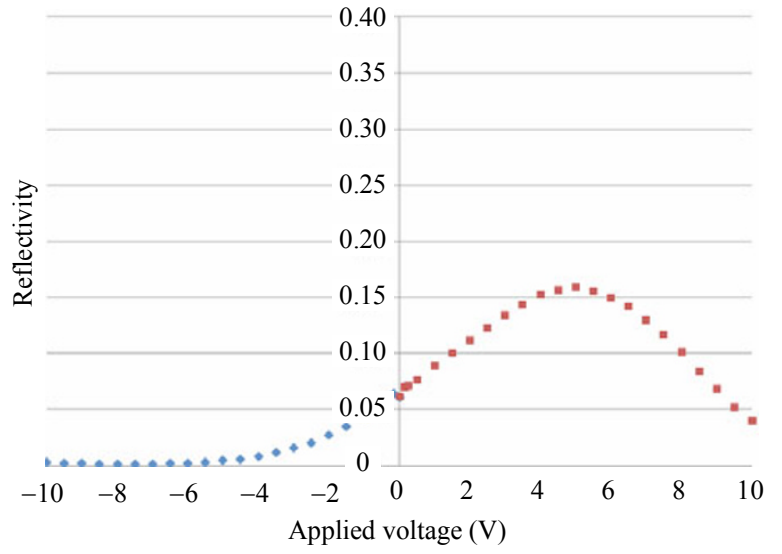

Fig. 7 Static response of the FLC cell for $\beta=12^{\circ}$ corresponding to the lowest total harmonic distortion.

Furthermore, potential devices based on these cells could make use of an optical fiber to deliver the incident light and collect the reflected light. In order to exploit the electro-optical properties of the liquid crystals, it would be necessary to use a polarization maintaining fibers, corresponding to a parallel polarizer/analyzer configuration. Noticing that, in our model, we have $R_{\perp}=1-R_{\|}$, we can easily see that all the above considerations, including the linear response, remain valid. This linearly connects the electro-optic response of the liquid crystal cell to a variation of the pressure making hydrophones based on such cells much simpler than other fiber optic sonar systems based on complicated demodulation schemes. 
The dynamic range is another important parameter of the sensing element. For each polarization angle, we thus measured the voltage corresponding to the maximum acceptable THD,

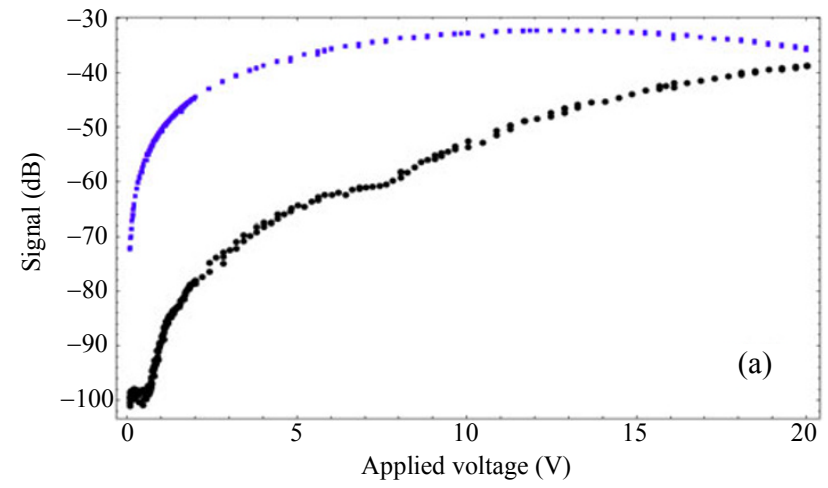

(a) which we set to be $-45 \mathrm{~dB}$. With reference to Fig. 5(b), we see that the largest acceptable voltages for the two angles $\beta=12^{\circ}$ and $14.5^{\circ}$ are $1.3 \mathrm{~V}$ and $0.4 \mathrm{~V}$, respectively.

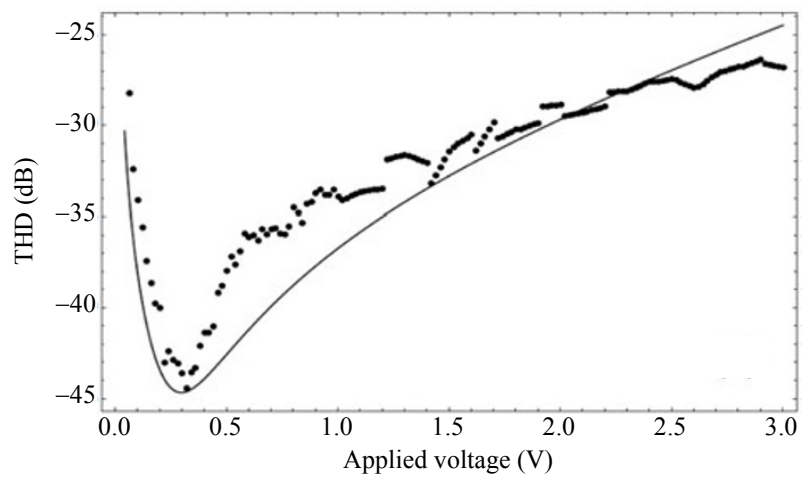

(b)

Fig. 8 Harmonics analysis: (a) intensity of the fundamental (1 kHz, top) and second (2 kHz, bottom) harmonics measured as a function of the applied voltage amplitude and (b) measured (symbols) and calculated (solid line) THDs plus noise vs. amplitude of the applied voltage at $\beta=12^{\circ}$.

Experimental data of the static response of the DHF for the bulk optic approach is presented in Fig. 7 for $\beta=12^{\circ}$, corresponding to the most linear response observed experimentally.

The Fourier analysis of the cell response operated at $1 \mathrm{kHz}$ is presented in Fig. 8. The first (signal) and second (ghost) harmonic intensities are plotted as a function of the applied voltage in Fig. 8(a). The ghost intensity stays at or below the $-100 \mathrm{~dB}$ level up to $0.3 \mathrm{~V}$, while the signal amplitude increases up to $-56 \mathrm{~dB}$ in the same range. This explains the initial drop in the THD illustrated in Fig. 8(b). Past this point, the ghost signal starts to increase faster than the signal itself causing the THD to increase. As a consequence, the THD goes through a minimum, which in our case is at a voltage of $0.3 \mathrm{~V}$. This behavior is well described by the theory when a noise $P_{N}>0$ is introduced [Fig. 8(b)].

The bandwidth of the cell was also investigated. The signal to noise ratio (SNR) as a function of the frequency is presented in Fig. 9 for an applied voltage amplitude of $0.25 \mathrm{~V}$, which corresponded to the THD of approximately $-45 \mathrm{~dB}$. At that operating voltage, the SNR dropped by $3 \mathrm{~dB}$ from $56 \mathrm{~dB}$ to $53 \mathrm{~dB}$ over the range of 0 to $5 \mathrm{kHz}$, which defined the bandwidth of the cell.

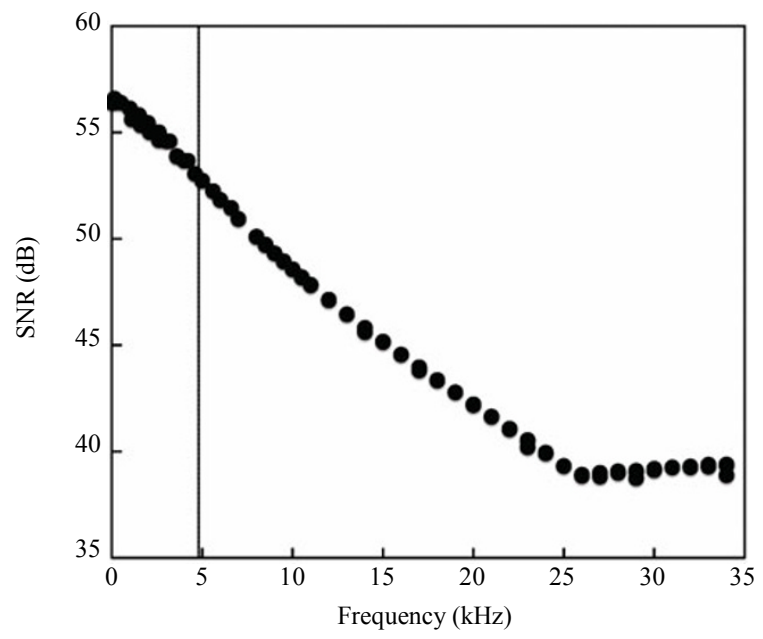

Fig. 9 SNR as a function of the applied voltage frequency.

A sensor head based on the proposed fiber optic approach was also characterized. The static response of the sensor head is presented in Fig. 10, where the angle between the polarization of incident light and the helix axis was adjusted to minimize the THD. The stability of the device was also estimated. The 
optical signal reflected off the cell was fluctuating by $0.44 \%$ for a measured applied voltage fluctuation of $\pm 0.03 \%$ (peak-to-peak amplitude of $19.6 \mathrm{~V}$ at the frequency of $50 \mathrm{~Hz})$.

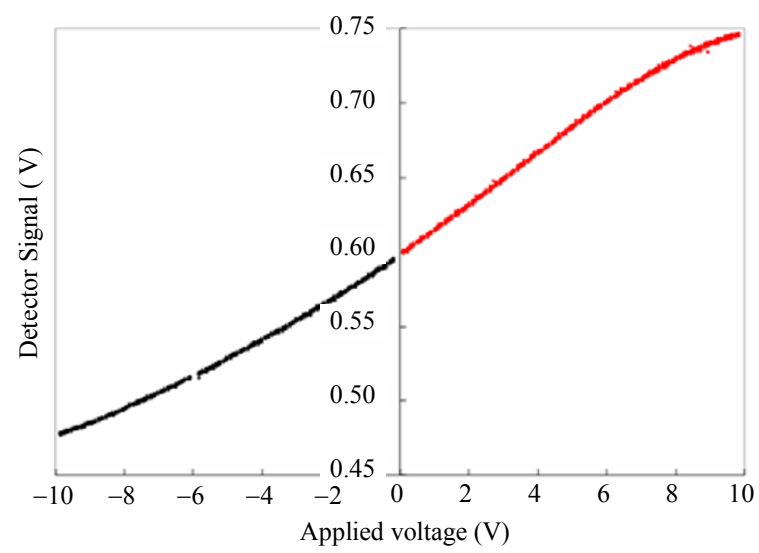

Fig. 10 Static response of the sensor head using a fiber-based approach.

\section{Conclusions}

A new low cost method for the optical measurement of sound under water at distributed localized points was proposed. This method uses a deformed helix ferroelectric liquid crystal (DHF-LC) cell combined with the PZT ceramic hydrophone as a sensor head for the optical measurement of the sound waves. The use of the ferroelectric liquid in the DHF mode exploited the linear electrooptic response of the liquid crystal cell to variation of the external electrical field. Such cells were built and fully characterized. The optimal parameters of the cell's operation in sensing applications were determined and experimentally measured while the performance of the SH using these parameters were quantified. The comparison of the theory with experimental results revealed excellent agreement.

\section{Acknowledgment}

This work was supported by the Australian government, Smart Digital Optics and The Hong Kong University of Science and Technology through the Australian Research Council (ARC), the
ARC-Linkage research funding program and HKUST grants CERG 612409 and CERG 612310. Russian Foundation of Basic Research Grants 10-02-01336-a, 10-03-13305-PT-omi， 11-02-92492 -MNTI_a are gratefully acknowledged.

Open Access This article is distributed under the terms of the Creative Commons Attribution License which permits any use, distribution, and reproduction in any medium, provided the original author(s) and source are credited.

\section{References}

[1] S. G. Lemon, "Towed-array history, 1917-2003," IEEE Journal of Oceanic Engineering, vol. 29, no. 2, pp. 365-373, 2004.

[2] J. A. Bucaro and H. D. Dardy, "Fiber-optic hydrophone," J. Acoust. Soc. Am., vol. 62, no. 5, pp. 1302-1303, 1977.

[3] G. Wild and S. Hinckley, "Acousto-ultrasonic optical fiber sensors: overview and state-of-the-art," IEEE Sensors Journal, vol. 8, no. 7, pp.1184-1193, 2008.

[4] K. T. V. Gratten and B. T. Meggitt, Optical Fiber Sensor Technology: Applications and systems. Dordrecht, The Netherlands: Kluwer Academic Publishers, 1999.

[5] I. Leung, Z. Brodzeli, T. Whitbread, X. Chen, and G. Peng, "A distributed-feedback fiber-laser-based optical fiber hydrophone system with very high sensitivity," in Proc. SPIE (Advanced Sensor Systems and Applications II), vol. 5634, pp. 434-443, 2005.

[6] S. Atique, D. Betz, B. Culshaw, F. Dong, H. S. Park, G. Thursby, et al., "Detecting ultrasound using optical fibres," J. Optics, vol. 33, no. 4, pp. 231-238, 2004.

[7] S. K. Sheem and J. H. Cole, "Acoustic sensitivity of single-mode optical power dividers," Optics Letters, vol. 4, no. 10, pp. 322-324, 1979.

[8] L. A. Beresnev, V. G. Chigrinov, D. I. Dergachev, E. P. Poshidaev, J. Fünfschilling, and M. Schadt, "Deformed helix ferroelectric liquid crystal display: a new electrooptic mode in ferroelectric chiral smectic C liquid crystals," Liquid Crystals, vol. 5, no. 4, pp. 1171-1177, 1989.

[9] B. I. Ostrovskii and V. G. Chigrinov, "Linear 
electrooptic effect in chiral smectic C liquid crystals," Kristallografiya, vol. 25, no. 3, pp. 560-567, 1980.

[10] V. G. Chigrinov, V. A. Baikalov, E. P. Pozhidaev, L. M. Blinov, L. A. Beresnev, and A. I. Allagulov, "Flexoelectric polarization of ferroelectric smectic liquid crystal," Zhurnal Eksperimentalnoi $i$ Teoreticheskoi Fiziki, vol. 88, no. 6, pp. 2015-2024, 1985.

[11] A. D. Kiselev, E. P. Pozhidaev, V. G. Chigrinov, and
H. S. Kwok, "Polarization-gratings approach to deformed-helix ferroelectric liquid crystals with subwavelength pitch," Physical Review E, vol. 83, no. 3, pp. 031703, 2011.

[12] E. P. Pozhidaev, V. G. Chigrinov, D. Huang, A. Zhukov, J. Ho, and H. S. Kwok, "Photoalignment of ferroelectric liquid crystals by azodye layers," Japanese Journal of Applied Physics, vol. 43, no. 8A, pp. 5440-5446, 2004. 